\section{On necessity and robustness of dissipativity in economic model predictive control}

\author{
Matthias A. Müller, David Angeli, and Frank Allgöwer
}

\begin{abstract}
In this paper, we study a dissipativity property which was recently used in several results on economic model predictive control to ensure optimal operation of a system at steady-state as well as stability. In particular, we first investigate whether this dissipativity property is not only sufficient, but also necessary for optimal steady-state operation. In the most general case, this is not true; nevertheless, under an additional controllability assumption, we show that dissipativity is in fact necessary. Second, we provide a robustness analysis of the dissipativity property with respect to changes in the constraint set, which can result in a change in the considered supply rate.
\end{abstract}

\section{INTRODUCTION}

In recent years, model predictive control (MPC) has become one of the most successful control strategies with an ever growing number of applications in various industries, thanks to its ability to satisfy hard state and input constraints and to directly incorporate a performance criterion. When designing an MPC controller, the underlying optimization problem is usually formulated as a tracking problem, i.e., the control objective is to track a certain (given) setpoint or trajectory the system has to follow (see, e.g., [2,3] and the references therein). This translates to requiring that the respective stage cost function is positive definite with respect to the considered setpoint or trajectory. In order to ensure certain theoretical properties of the closed-loop system such as stability and robustness, different MPC schemes have been proposed in the literature, either with [2] or without [3] additional (terminal) constraints and/or cost terms.

However, the above mentioned basic assumption that the stage cost function is positive definite with respect to a given target set, need not be satisfied in general. Hence recently, a more general MPC framework has been introduced where this assumption is not needed, which was termed economic MPC [4]. The wording is due to the fact that such a setup was motivated and is in particular useful when optimizing process economics, where the assumption of positive definiteness of the cost is not satisfied (for some recent examples, see, e.g., [5-7]). Again, different economic MPC formulations either with [4, 6, 8-12] or without [13] additional (terminal) constraints have been proposed and analyzed recently. One of the key features of economic MPC is that due to the use of a general cost function, the resulting closed-loop system is not necessarily convergent. Hence one of the key questions is what an optimal trajectory looks like, e.g., whether operation of the system at some steady-state or some periodic behavior is optimal in the sense that no other feasible trajectory leads to a smaller (average) cost. Furthermore, in case that steadystate operation is optimal, it is desirable that the closed-loop system resulting from application of an economic MPC algorithm does in fact converge to the optimal steady-state. For both of the above questions, a certain dissipativity condition has turned out to play a crucial role $[4,9-11,13]$. Namely, in these references it is assumed that a

A preliminary version of parts of this paper has been presented at the 51st IEEE Conference on Decision and Control (CDC) 2012, see [1].

Matthias A. Müller and Frank Allgöwer are with the Institute for Systems Theory and Automatic Control, University of Stuttgart, 70550 Stuttgart, Germany. Their work was supported by the German Research Foundation (DFG) within the Priority Programme 1305 "Control Theory of Digitally Networked Dynamical Systems" and within the Cluster of Excellence in Simulation Technology (EXC 310/1) at the University of Stuttgart. \{mueller, allgower\}@ist.uni-stuttgart.de

David Angeli is with the Department of Electrical and Electronic Engineering, Imperial College, London, UK and Dip. di Ingegneria dell'Informazione, University of Firenze, Italy. d.angeli@imperial.ac.uk storage function $\lambda$ exists such that the considered open-loop system is (strictly) dissipative with respect to a supply rate $s$ depending on the stage cost function and also the constraint set, in terms of the optimal feasible steady-state (see Section II-B for a more detailed setup). This dissipativity condition was then shown to be sufficient for optimal steady-state operation of a system [4,9] and it was also used for convergence and stability analysis $[4,10,11,13]$.

The goal of this paper is to thoroughly investigate and provide further insight into the mentioned crucial dissipativity condition used in economic MPC. In particular, the following two main contributions are obtained. First, we examine whether this dissipativity condition is not only sufficient, but also necessary for a system to be optimally operated at steady-state (see Section III). While in the most general case, this turns out not to be true, we show that if an additional controllability/reachability condition is satisfied, dissipativity is in fact necessary for optimal steady-state operation. The second contribution of this paper (see Section IV) is to provide a robustness analysis of the mentioned dissipativity condition with respect to changes in the constraint set. Namely, as noted above, the supply rate used in economic MPC depends on the optimal feasible steady-state (and hence on the constraints imposed on the system), and thus dissipativity might be lost if the constraints are changed. In Section IV-A, it is shown that under certain assumptions, robustness of the dissipativity condition with respect to small changes in the constraint set can be ensured. Moreover, if a certain convexity assumption is satisfied, then further results on maintaining the dissipativity property in addition to the presented robustness analysis can be obtained, which will be shown in Section IV-B. Finally, we remark that the proofs of some of the following results are not included in this technical note, but can be found online in the technical report [14].

\section{Preliminaries AND SETUP}

\section{A. Notation}

Let $\mathbb{I}_{[a, b]}$ denote the set of integers in the interval $[a, b] \subseteq \mathbb{R}$, and $\mathbb{I}_{\geq a}$ the set of integers greater than or equal to $a$. For $a \in \mathbb{R},\lceil a\rceil$ is defined as the smallest integer greater than or equal to $a$. As in [4], for any vector valued bounded signal $v: \mathbb{I}_{\geq 0} \rightarrow \mathbb{R}^{n_{v}}$ we define the set of asymptotic averages as

$$
A v[v]:=\left\{\bar{v} \in \mathbb{R}^{n_{v}}: \exists t_{n} \rightarrow+\infty: \lim _{n \rightarrow \infty} \frac{\sum_{k=0}^{t_{n}} v(k)}{t_{n}+1}=\bar{v}\right\} .
$$

Note that $A v[v]$ is nonempty (as bounded sequences in $\mathbb{R}^{n_{v}}$ have limit points), but it need not be a singleton in general.

\section{B. Problem setup - dissipativity in economic model predictive control}

We consider discrete-time nonlinear systems of the form

$$
x(k+1)=f(x(k), u(k)), \quad x(0)=x_{0},
$$

with $k \in \mathbb{I}_{>0}$, where $x \in \mathbb{X} \subseteq \mathbb{R}^{n}$ and $u \in \mathbb{U} \subseteq \mathbb{R}^{m}$. We assume that $f$ is continuous in $(x, u)$. The system is subject to (possibly coupled) pointwise-in-time state and input constraints $(x(k), u(k)) \in \mathbb{Z}, k \in$ $\mathbb{I}_{>0}$, for some compact set $\mathbb{Z} \subseteq \mathbb{X} \times \mathbb{U}$. Define the set $\mathbb{Z}^{0}$ as the largest "forward invariant" set contained in $\mathbb{Z}$, i.e., the set which contains all elements in $\mathbb{Z}$ which are part of a feasible trajectory $(\mathbf{z}, \mathbf{v})$ :

$$
\begin{aligned}
\mathbb{Z}^{0} & :=\{(x, u) \in \mathbb{Z}: \exists \mathbf{v} \text { s.t. }(z(0), v(0))=(x, u), \\
& \left.z^{+}=f(z, v),(z(k), v(k)) \in \mathbb{Z} \forall k \in \mathbb{I}_{\geq 0},\right\} \subseteq \mathbb{Z} .
\end{aligned}
$$

Denote by $\mathbb{X}^{0}$ the projection of $\mathbb{Z}^{0}$ on $\mathbb{X}$, i.e., $\mathbb{X}^{0}:=\{x \in \mathbb{X}: \exists u \in$ $\mathbb{U}$ s.t. $\left.(x, u) \in \mathbb{Z}^{0}\right\}$. Furthermore, let $S$ be defined as the set of all feasible steady-states of system (2), i.e., $S:=\{(x, u) \in \mathbb{Z}: x=$ $f(x, u)\}$, which is assumed to be non-empty. 
In order to compute a control input to system (2) in a model predictive control framework, the system (2) is equipped with a stage cost $\ell: \mathbb{R}^{n} \times \mathbb{R}^{m} \rightarrow \mathbb{R}$ which is assumed to be continuous. The control input is then calculated by minimizing, at each time instant $k$, a cost function

$$
J(x, \mathbf{u})=\sum_{i=k}^{k+T} \ell(x(i \mid k), u(i \mid k))
$$

over the finite horizon $T$ subject to the state and input constraints, where $\mathbf{u}:=[u(k \mid k), \ldots, u(k+T \mid k)]$ and $\mathbf{x}:=[x(k \mid k), \ldots, x(k+$ $T \mid k)$ ] (with initial condition $x(k \mid k)=x(k)$ ) denote the predicted input and corresponding state sequences, respectively. Then, the first part of the optimal input sequence is applied to system (2) in a receding horizon fashion.

Remark 1: In certain economic MPC settings in the literature, a terminal cost term and/or a terminal constraint are added to (4) (see, e.g, $[4,6,10,11])$, while other schemes do not use such additional ingredients [13]. The results which will be developed in this paper are important and find application in both such settings with and without additional terminal constraints and/or cost terms. Namely, the dissipativity condition which we examine plays a crucial role in both frameworks, and it is formulated independent of the specific economic MPC setting.

As explained in Section I, the stage cost $\ell$ in (4) can be a general, possibly economic, cost function and need not be positive definite with respect to any setpoint. We now define the set $S^{*}$ as the set of all feasible steady-states which are optimal with respect to the stage cost $\ell$, i.e.,

$$
S^{*}:=\left\{(y, w) \in S: \ell(y, w)=\min _{(x, u) \in S} \ell(x, u)\right\} .
$$

Note that $S^{*}$ is non-empty and well defined, i.e., the minimum in (5) exists as $S$ is compact and $\ell$ is continuous. In general, $S^{*}$ need not be a singleton, i.e., there does not necessarily exist a unique optimal feasible steady-state $\left(x^{*}, u^{*}\right)$. In the following, by $\left(x^{*}, u^{*}\right)$ we denote an arbitrary element of the set $S^{*}$. As in [4, Definition 6.1], we now define optimal operation of a system at steady-state as follows:

Definition 1: The system (2) is optimally operated at steady-state with respect to the cost function $\ell$, if for each solution satisfying $(x(k), u(k)) \in \mathbb{Z}$ for all $k \in \mathbb{I}_{\geq 0}$ the following holds:

$$
A v[\ell(x, u)] \subseteq\left[\ell\left(x^{*}, u^{*}\right), \infty\right),
$$

where $\left(x^{*}, u^{*}\right) \in S^{*}$ is an optimal steady-state as defined via (5). The system (2) is suboptimally operated off steady-state, if in addition at least one of the following two conditions holds:

$$
\begin{array}{r}
A v[\ell(x, u)] \subseteq\left(\ell\left(x^{*}, u^{*}\right), \infty\right) \\
\liminf _{t \rightarrow \infty}\left|x(t)-x^{*}\right|=0
\end{array}
$$

Furthermore, when examining necessity in Section III, we need the slightly stricter definition of uniform suboptimal operation off steady-state:

Definition 2: The system (2) is uniformly suboptimally operated off steady-state, if it is suboptimally operated off steady-state and in addition for each $\delta$, there exists $\bar{t} \in \mathbb{I}_{\geq 1}$ such that for each feasible solution at least one of the following two conditions holds:

$$
\begin{aligned}
\sum_{k=0}^{t-1} \frac{\ell(x(k), u(k))}{t} \geq \ell\left(x^{*}, u^{*}\right) & \text { for all } t \geq \bar{t} \\
\left|x(s)-x^{*}\right| \leq \delta & \text { for some } s \in \mathbb{I}_{[1, \bar{t}]}
\end{aligned}
$$

Remark 2: The definition of optimal operation at steady-state is such that no feasible solution to system (2) leads to an average performance (measured in terms of the stage cost $\ell$ ) which is better than operation of the system at the optimal steady-state $\left(x^{*}, u^{*}\right)$.
In the definition of uniform suboptimal operation off steady-state, uniformity is with respect to all initial conditions and feasible sequences. Namely, each feasible sequence either passes by arbitrarily close at the optimal steady state within the finite time interval $[1, \bar{t}]$ (which only depends on the distance $\delta$ from $x^{*}$, but not on the specific sequence) or has a transient performance greater or equal to steadystate performance for all $t \geq \bar{t}$.

In order to study optimal steady-state operation of a system, a certain dissipativity condition was used in $[4,9,13]$. The notion of dissipativity was introduced in [15] (for a discrete time version see [16]); we adapt it here to our setting including state and input constraints. To this end, for a set $\mathbb{W} \subseteq \mathbb{Z}$, denote by $\mathbb{W}_{\mathbb{X}}$ the projection of $\mathbb{W}$ on $\mathbb{X}$, i.e., $\mathbb{W}_{\mathbb{X}}:=\{x \in \mathbb{X}: \exists u \in \mathbb{U}$ s.t. $(x, u) \in$ $\mathbb{W}\}$.

Definition 3: The system (2) is dissipative on a set $\mathbb{W} \subseteq \mathbb{Z}$ with respect to the supply rate $s: \mathbb{X} \times \mathbb{U} \rightarrow \mathbb{R}$ if there exists a bounded storage function $\lambda: \mathbb{W}_{\mathbb{X}} \rightarrow \mathbb{R}$ such that the following inequality is satisfied for all $(x, u) \in \mathbb{W}$ :

$$
\lambda(f(x, u))-\lambda(x) \leq s(x, u) .
$$

If, in addition, for some positive definite ${ }^{1} \rho: \mathbb{W}_{\mathbb{X}} \rightarrow \mathbb{R}_{\geq 0}$ it holds that for all $(x, u) \in \mathbb{W}$

$$
\lambda(f(x, u))-\lambda(x) \leq-\rho(x)+s(x, u),
$$

then system (2) is strictly dissipative on $\mathbb{W}$.

Note that in the original definition $[15,16]$, the storage function $\lambda$ is required to be nonnegative; in accordance with [4,9-11,13], we do not impose this assumption here but only require that it is bounded on the bounded $\operatorname{set}^{2} \mathbb{W}_{\mathbb{X}}$. We can now state the following result relating dissipativity of system (2) with optimal steady-state operation, which is a slight extension of [4, Proposition 6.4].

Theorem 1: Suppose that system (2) is dissipative (strictly dissipative) on $\mathbb{Z}^{0}$ with respect to the supply rate $s(x, u):=\ell(x, u)-$ $\ell\left(x^{*}, u^{*}\right)$. Then the system (2) is optimally operated at steady-state (uniformly suboptimally operated off steady-state).

Proof: Sufficiency of the above dissipativity (strict dissipativity) condition for optimal steady state operation (suboptimal operation off steady-state) was shown in [4, Proposition 6.4]. Hence it remains to show that strict dissipativity results in uniform suboptimal operation off steady-state. From strict dissipativity (see (10)), it follows that for each feasible solution and each $t \in \mathbb{I}_{\geq 0}$

$$
\begin{aligned}
-c & :=-2 \sup _{x \in \mathbb{X}^{0}}|\lambda(x)| \leq \lambda(x(t))-\lambda(x(0)) \\
& \leq \sum_{k=0}^{T-1}\left[\ell(x(k), u(k))-\ell\left(x^{*}, u^{*}\right)-\rho(x(k))\right] .
\end{aligned}
$$

As $\rho$ is positive definite with respect to $x^{*}$, there exists a function $\hat{\rho} \in$ $\mathcal{K}_{\infty}$ such that $\rho(x) \geq \hat{\rho}\left(\left|x-x^{*}\right|\right)$ for all $x \in \mathbb{X}^{0}$. Let $\delta>0$ be arbitrary but fixed, and define $\bar{t}:=\lceil c / \hat{\rho}(\delta)\rceil+1$. Then, from (11) it directly follows that either $\sum_{k=0}^{t-1} \ell(x(k), u(k)) \geq t \ell\left(x^{*}, u^{*}\right)$ for all $t \geq \bar{t}$ (and hence also (8a) is satisfied), or $\left|x(s)-x^{*}\right| \leq \delta$ for at least two time instants $s \in \mathbb{I}_{[0, \bar{t}]}$ and hence for at least one time instant $s \in \mathbb{I}_{[1, \bar{t}]}$. Namely, if both of the above were not true, i.e., $\sum_{k=0}^{t-1} \ell(x(k), u(k))<t \ell\left(x^{*}, u^{*}\right)$ for at least one time instant $t \geq \bar{t}$ and $\left|x(s)-x^{*}\right| \leq \delta$ for at most one time instant $s \in \mathbb{I}_{[0, \bar{t}]}$, one

${ }^{1} \mathrm{~A}$ function $\rho$ is positive definite with respect to some point $\bar{x} \in \mathbb{X}$ if it is continuous, $\rho(\bar{x})=0$ and $\rho(x)>0$ for all $x \in \mathbb{X}$ with $x \neq \bar{x}$. In the following, when speaking of strict dissipativity, we take $\bar{x}=x^{*}$, i.e., the function $\rho$ is assumed to be positive definite with respect to the optimal steady-state $x^{*}$ defined via (5).

${ }^{2}$ In fact, one could then also just add a constant to make $\lambda$ nonnegative. 
would obtain that

$$
\begin{aligned}
& \sum_{k=0}^{t-1}\left[\ell(x(k), u(k))-\ell\left(x^{*}, u^{*}\right)-\rho(x(k))\right] \\
& \leq-(\bar{t}-1) \hat{\rho}(\delta)+\sum_{k=0}^{t-1}\left[\ell(x(k), u(k))-\ell\left(x^{*}, u^{*}\right)\right] \\
& <-(\bar{t}-1) \hat{\rho}(\delta)=-\lceil c / \hat{\rho}(\delta)\rceil \hat{\rho}(\delta) \leq-c,
\end{aligned}
$$

which contradicts (11). Hence we conclude that system (2) is uniformly suboptimally operated off steady-state.

Remark 3: When actually searching for a storage function $\lambda$ for a given system and constraint sets, one might rather consider the possibly larger set $\mathbb{Z}$ instead of $\mathbb{Z}^{0}$ and establish dissipativity there, as $\mathbb{Z}^{0}$ is in general difficult to compute. We will take this approach in Section IV, where we describe the set $\mathbb{Z}$ via inequality constraints. However, the results presented there can analogously be stated with $\mathbb{Z}^{0}$ instead, if this set can also be described by inequalities.

Besides being sufficient for optimal steady-state operation, the strict dissipativity assumption of Theorem 1 can furthermore be used for stability analysis in the context of economic MPC with $[4,11]$ and without terminal constraints [13]. Now define the function

$$
\gamma(x, u):=\ell(x, u)-\ell\left(x^{*}, u^{*}\right)+\lambda(x)-\lambda(f(x, u)) .
$$

The dissipativity assumption of Theorem 1 implies that for all $(x, u) \in \mathbb{Z}^{0}, \gamma(x, u) \geq 0$ in case of dissipativity and $\gamma(x, u) \geq$ $\alpha\left(\left|x-x^{*}\right|\right)$ for some $\alpha \in \mathcal{K}_{\infty}$ in case of strict dissipativity. In Section IV, in some places we will use a slightly stronger assumption than strict dissipativity, namely that $\left(x^{*}, u^{*}\right)$ is a unique minimizer of $\gamma(x, u)$ on $\mathbb{Z}$, i.e., there exists a function $\alpha \in \mathcal{K}_{\infty}$ such that $\gamma(x, u) \geq \alpha\left(\left|\left(x-x^{*}, u-u^{*}\right)\right|\right)$ for all $(x, u) \in \mathbb{Z}$.

In the following, we will more closely examine the dissipativity condition of Theorem 1. In Section III, we discuss whether it is not only sufficient, but also necessary for steady-state optimality, before examining robustness properties with respect to changes in the constraint sets (and hence in the supply rate $s$ ) in Section IV.

\section{NECESSITY OF DISSIPATIVITY FOR OPTIMAL STEADY-STATE OPERATION}

In this section, we examine under what conditions the dissipativity condition in Theorem 1 is not only sufficient, but also necessary for optimal operation of system (2) at steady-state. In the most general case, it turns out that this is not true, as we showed in our previous work [17] by means of two counterexamples, in which a system was optimally operated at steady-state but not dissipative with respect to the supply rate $s(x, u)=\ell(x, u)-\ell\left(x^{*}, u^{*}\right)$. Nevertheless, under an additional controllability assumption on the system, in the following we obtain two (partial) converse results of Theorem 1 concerning necessity of dissipativity for optimal steady-state operation and uniform suboptimal operation off steady-state, respectively. We first recall the definition of available storage from [15, Definition 3] and slightly adapt it to our setting with state and input constraints.

Definition 4: For a given supply rate $s: \mathbb{Z}^{0} \rightarrow \mathbb{R}$ and each $x \in$ $\mathbb{X}^{0}$, the available storage $S_{a}$ of system (2) subject to state and input constraints $(x, u) \in \mathbb{Z}^{0}$ is defined as

$$
S_{a}(x):=\sup _{\substack{T \geq 0 \\ z(0)=x, z(k+1)=f(z(k), v(k)) \\(z(k), v(k)) \in \mathbb{Z}^{0} \quad \forall k \in \mathbb{I} \geq 0}} \sum_{k=0}^{T-1}-s(z(k), v(k))
$$

Note that $S_{a}(x)$ is nonnegative for all $x \in \mathbb{X}^{0}$, as $T=0$ is allowed in (13) and by convention the empty sum is zero. In [15], it was shown that the available storage plays a crucial role for establishing dissipativity of a system.

Theorem 2 ([15, Theorem 1]): System (2) is dissipative on $\mathbb{Z}^{0}$ with respect to the supply rate $s$ if and only if the available storage $S_{a}$ defined in (13) is bounded on $\mathbb{X}^{0}$. Moreover, $S_{a}$ is a storage function according to Definition 3, Equation (9).

Remark 4: The original proof in [15] was done for continuoustime systems without state and input constraints; however, it can straightforwardly be adapted to our setting of discrete-time systems with state and input constraints. Furthermore, as already mentioned above, the definition of dissipativity in [15] is such that the storage function $\lambda$ is required to be nonnegative, while we require that it is bounded on $\mathbb{X}^{0}$. Again, the proof in [15] can straightforwardly be adapted to this modified setting.

\section{A. Necessity of dissipativity under a controllability / reachability condition}

In this section, we show that dissipativity with respect to the supply rate $s(x, u)=\ell(x, u)-\ell\left(x^{*}, u^{*}\right)$ is in fact necessary for optimal steady-state operation under a certain controllability/reachability condition. To this end, we need the following definitions. For a given $N \in \mathbb{I}_{\geq 1}$, denote by $\mathcal{X}_{N} \subseteq \mathbb{X}$ the set of states which can be steered to the optimal steady-state $x^{*}$ in $N$ steps in a feasible way, i.e.,

$$
\begin{gathered}
\mathcal{X}_{N}:=\left\{x \in \mathbb{X}: \exists \mathbf{v} \text { s.t. } z(0)=x, z^{+}=f(z, v), z(N)=x^{*},\right. \\
\left.(z(k), v(k)) \in \mathbb{Z} \forall k \in \mathbb{I}_{[0, N-1]}\right\} .
\end{gathered}
$$

Next, let $\mathcal{R}_{N}$ be the set of states which can be reached from the optimal steady-state $x^{*}$ in $N$ steps in a feasible way, i.e.,

$$
\begin{gathered}
\mathcal{R}_{N}:=\left\{x \in \mathbb{X}: \exists \mathbf{v} \text { s.t. } z(0)=x^{*}, z^{+}=f(z, v), z(N)=x,\right. \\
\left.(z(k), v(k)) \in \mathbb{Z} \forall k \in \mathbb{I}_{[0, N-1]}\right\} .
\end{gathered}
$$

Note that $\mathcal{X}_{N} \cap \mathcal{R}_{N} \neq \emptyset$, as by definition $x^{*}$ is contained in both $\mathcal{X}_{N}$ and $\mathcal{R}_{N}$. Now define the set $\mathbb{Z}_{N}$ as the set of state/input pairs which are part of a feasible state/input sequence pair $(\mathbf{z}, \mathbf{v})$ which is such that $z(\cdot)$ stays in the intersection of $\mathcal{X}_{N}$ and $\mathcal{R}_{N}$ for all times:

$$
\begin{aligned}
\mathbb{Z}_{N}:= & \left\{(x, u) \in \mathbb{Z}: \exists \mathbf{v} \text { s.t. }(z(0), v(0))=(x, u), z^{+}=f(z, v),\right. \\
& \left.(z(k), v(k)) \in \mathbb{Z}, z(k) \in \mathcal{X}_{N} \cap \mathcal{R}_{N} \forall k \in \mathbb{I}_{\geq 0},\right\} \subseteq \mathbb{Z}^{0} .
\end{aligned}
$$

Finally, denote the projection of $\mathbb{Z}_{N}$ on $\mathbb{X}$ by $\mathbb{X}_{N}$, i.e.,

$$
\mathbb{X}_{N}:=\left\{x \in \mathbb{X}: \exists u \in \mathbb{U} \text { s.t. }(x, u) \in \mathbb{Z}_{N}\right\} .
$$

Note that $\mathbb{X}_{N} \subseteq \mathcal{X}_{N} \cap \mathcal{R}_{N} \subseteq \mathbb{X}_{2 N}$, where the first inequality directly follows from the definition of $\mathbb{Z}_{N}$ and the second follows from the fact that for each $y \in \mathcal{X}_{N} \cap \mathcal{R}_{N}$, there exist a feasible state sequence with $x(0)=x^{*}, x(N)=y$, and $x(2 N)=x^{*}$, which implies that $y \in \mathbb{X}_{2 N}$. We can now state the following result concerning necessity of dissipativity for optimal steady-state operation.

Theorem 3: Suppose that system (2) is optimally operated at steady-state. Then, for each $N \in \mathbb{I}_{\geq 1}$, system (2) is dissipative on $\mathbb{Z}_{N}$ with respect to the supply rate $s(x, u):=\ell(x, u)-\ell\left(x^{*}, u^{*}\right)$.

Proof: Fix an arbitrary $N \in \mathbb{I}_{\geq 1}$. For simplicity and without loss of generality, in the following we assume that $\ell\left(x^{*}, u^{*}\right)=0$. Assume for contradiction that the system is optimally operated at steady-state, but it is not dissipative on $\mathbb{Z}_{N}$. Applying Theorem 2 with $\mathbb{Z}^{0}$ and $\mathbb{X}^{0}$ replaced by $\mathbb{Z}_{N}$ and $\mathbb{X}_{N}$, respectively, it follows that this is equivalent to the fact that the available storage (see Definition 4 with $\mathbb{Z}^{0}$ replaced by $\mathbb{Z}_{N}$ ) is unbounded on $\mathbb{X}_{N}$, and hence for each $r \geq 0$ there exists 
some $y \in \mathbb{X}_{N}$ such that

$$
\inf _{\substack{T \geq 0 \\ z(0)=y \\=f(z(k), v(k)) \\ k)) \in \mathbb{Z}_{N}}} \sum_{\substack{k=0 \\ \forall-\mathbb{I}_{\geq 0}}}^{T-1} \ell(z(k), v(k)) \leq-r .
$$

This means that for each $r \geq 0$, there exist some $y \in \mathbb{X}_{N}$ and a state/input sequence pair $x_{r}(\cdot), u_{r}(\cdot)$ together with a time instant $T_{r} \in \mathbb{I}_{\geq 0}$, such that $x_{r}(0)=y,\left(x_{r}(k), u_{r}(k)\right) \in \mathbb{Z}_{N}$ for all $k \in \mathbb{I}_{\geq 0}$ and

$$
\sum_{k=0}^{T_{r}-1} \ell\left(x_{r}(k), u_{r}(k)\right) \leq-r .
$$

Now fix some $r \geq 1+2 N \max _{(x, u) \in \mathbb{Z}} \ell(x, u)$. By definition of $\mathbb{Z}_{N}$, we have $x_{r}\left(T_{r}\right) \in \mathcal{R}_{N} \cap \mathcal{X}_{N}$. Hence there exists a state/input sequence pair $x^{\prime}(\cdot), u^{\prime}(\cdot)$ satisfying $x^{\prime}(0)=x_{r}\left(T_{r}\right), x^{\prime}(N)=x^{*}$, $x^{\prime}(2 N)=x_{r}(0)=y$ and $\left(x^{\prime}(t), u^{\prime}(t)\right) \in \mathbb{Z}$ for all $t \in \mathbb{I}_{[0,2 N]}$.

Now define the following input sequence:

$$
\hat{u}\left(k\left(T_{r}+2 N\right)+i\right)= \begin{cases}u_{r}(i) & k \in \mathbb{I}_{\geq 0}, i \in \mathbb{I}_{\left[0, T_{r}-1\right]} \\ u^{\prime}(i) & k \in \mathbb{I}_{\geq 0}, i \in \mathbb{I}_{\left[T_{r}, T_{r}+2 N-1\right]}\end{cases}
$$

which results in a cyclic state sequence with $\hat{x}\left(k\left(T_{r}+2 N\right)\right)=y$ for all $k \in \mathbb{I}_{\geq 0}$. This state/input sequence pair fulfills $(\hat{x}(t), \hat{u}(t)) \in \mathbb{Z}$ for all $t \in \mathbb{I}_{>0}$ by construction, and furthermore we obtain for all $k \in \mathbb{I}_{\geq 0}$ :

$$
\begin{aligned}
& \sum_{i=0}^{T_{r}+2 N-1} \ell\left(\hat{x}\left(k\left(T_{r}+2 N\right)+i\right), \hat{u}\left(k\left(T_{r}+2 N\right)+i\right)\right) \\
& \stackrel{(20)}{=} \sum_{i=0}^{T_{r}-1} \ell(\hat{x}(i), \hat{u}(i))+\sum_{i=0}^{2 N-1} \ell\left(x^{\prime}(i), u^{\prime}(i)\right) \\
& \stackrel{(19)}{\leq}-r+2 N \max _{(x, u) \in \mathbb{Z}} \ell(x, u) \leq-1 .
\end{aligned}
$$

But this implies that

$$
\begin{aligned}
& \liminf _{T \rightarrow \infty} \sum_{k=0}^{T-1} \frac{\ell(\hat{x}(k), \hat{u}(k))}{T} \\
& \stackrel{(20)}{=} \frac{1}{T_{r}+2 N} \sum_{i=0}^{T_{r}+2 N-1} \ell(\hat{x}(i), \hat{u}(i)) \leq-\frac{1}{T_{r}+2 N}<0
\end{aligned}
$$

contradicting (6), i.e., optimal steady-state operation. Hence we conclude that the system (2) is dissipative on $\mathbb{Z}_{N}$ with respect to the supply rate $s(x, u):=\ell(x, u)-\ell\left(x^{*}, u^{*}\right)$.

Combining Theorems 1 and 3, we arrive at the following corollary.

Corollary 1: Suppose that $\mathbb{Z}_{N}=\mathbb{Z}^{0}$ for some $N \in \mathbb{I}_{\geq 1}$. Then system (2) is optimally operated at steady-state if and only if it is dissipative on $\mathbb{Z}^{0}$.

Remark 5: The results of Theorem 3 are still valid for a slightly different definition of $\mathbb{Z}_{N}$. Namely, in (16), the sets $\mathcal{X}_{N}$ and $\mathcal{R}_{N}$ can also be defined by replacing $x^{*}$ in (14) and (15), respectively, with any other state $y \in \mathbb{X}$.

\section{B. Necessity of dissipativity under a local controllability condition}

It is easy to show that $\mathbb{Z}_{N}=\mathbb{Z}^{0}$ for some $N \in \mathbb{I}_{\geq 1}$ if and only if $\mathbb{X}_{N}=\mathbb{X}^{0}$ for some $N \in \mathbb{I}_{\geq 1}$, which means that the system is weakly reversible (see $\left[18\right.$, Section 4.3]) in $\mathbb{X}^{0}$, i.e., each $x \in \mathbb{X}^{0}$ can be reached from and controlled to the optimal steady-state $x^{*}$ in a finite number of steps. In case that this reversibility condition is not satisfied, Theorem 3 only provides a partial converse result in the sense that dissipativity can only be ensured on a subset of $\mathbb{Z}^{0}$. In the following, we show that if a system is not only optimally operated at steady-state, but uniformly suboptimally operated off steady-state, then this reversibility condition is not needed in order to establish necessity of dissipativity on $\mathbb{Z}^{0}$, but a local controllability condition is enough. To this end, we assume that the function $f$ is once continuously differentiable in $(x, u)$ at $\left(x^{*}, u^{*}\right)$, i.e., the linearization of $f$ at $\left(x^{*}, u^{*}\right)$ exists.

Theorem 4: Suppose that system (2) is uniformly suboptimally operated off steady-state, that the linearization of (2) at the optimal steady-state $\left(x^{*}, u^{*}\right)$ is controllable, and that $\left(x^{*}, u^{*}\right) \in \operatorname{int}(\mathbb{Z})$. Then, system (2) is dissipative on $\mathbb{Z}^{0}$ with respect to the supply rate $s(x, u):=\ell(x, u)-\ell\left(x^{*}, u^{*}\right)$.

Proof: The proof of Theorem 4 can be found in Section I of the technical report [14].

Remark 6: The proof of Theorem 4 reveals that the obtained result is still correct if we assume the system to be locally controllable at the optimal steady-state instead of imposing the (in general stricter [18, Section 3.7]) requirement that the linearization is controllable. However, note that the latter is an easily verifiable condition while the former is, in general, not.

In summary, the results of Section III reveal that dissipativity with respect to the supply rate $s(x, u)=\ell(x, u)-\ell\left(x^{*}, u^{*}\right)$ is not only sufficient, but also necessary for optimal steady-state operation if a certain controllability condition is satisfied, and hence a lack of dissipativity despite optimal steady-state operation such as in the examples in [17] can only occur if the system exhibits some uncontrollable behavior. Furthermore, we note that the results presented in this section are also of importance in the context of stability and convergence analysis of economic MPC, for which the same (strict) dissipativity condition as in Theorem 1 is typically used [4, $10,11,13]$. Namely, while for linear systems with convex constraints and cost function, methods exist how a storage function $\lambda$ can be determined $[10,19]$, for general nonlinear systems with nonconvex constraints and cost function this can be a difficult task and, to the best of the authors' knowledge, no systematic procedure exists. Yet, in the above references, existence of a storage function $\lambda$ is sufficient for the stability analysis of the closed-loop system, but $\lambda$ does not have to be known for implementing the economic MPC algorithm, and Theorems 3 and 4 guarantee the existence of such a storage function $\lambda$ based on certain dynamic properties (controllability and optimal steady-state operation) of the considered system.

\section{ROBUSTNESS OF DISSIPATIVITY UNDER CHANGING CONSTRAINT SETS}

The supply rate of interest in economic MPC, $s(x, u)=\ell(x, u)-$ $\ell\left(x^{*}, u^{*}\right)$, depends on the state and input constraints which act on the system, namely through the optimal steady-state $\left(x^{*}, u^{*}\right)$. In this section, we examine what happens if the constraints are changed, and hence also the supply rate is altered. In general, dissipativity of system (2) with respect to the above supply rate may be lost even for arbitrarily small changes in the constraint set (see [1, Section III] for a simple example of this fact). In the following, we give conditions under which such a situation cannot occur. In particular, we first provide a robustness analysis of the considered dissipativity property with respect to small changes in the constraints, and then show that further results are possible if a certain convexity assumption is satisfied. To this end, in the following we consider several different optimization problems $\mathcal{P}\left(y, f_{0}, h, g\right)$ of the form

$$
\begin{aligned}
& \underset{y}{\operatorname{minimize}} f_{0}(y) \\
& \text { subject to } h(y)=0, g(y) \leq 0,
\end{aligned}
$$

with $y \in \mathbb{R}^{n_{y}}$ and some functions $f_{0}: \mathbb{R}^{n_{y}} \rightarrow \mathbb{R}, h: \mathbb{R}^{n_{y}} \rightarrow \mathbb{R}^{n_{h}}$ and $g: \mathbb{R}^{n_{y}} \rightarrow \mathbb{R}^{n_{g}}$. 


\section{A. Robustness of dissipativity with respect to changing supply rate}

In this section, we establish robustness of the considered dissipativity property with respect small changes in constraints. To this end, in the following we assume that the state and input constraint set $\mathbb{Z}$ is given in the form of inequality constraints, which depend on additional parameters $\varepsilon \in \mathbb{R}^{s}$, i.e.,

$$
\mathbb{Z}_{\varepsilon}:=\{(x, u): g(x, u ; \varepsilon) \leq 0\}
$$

for some function $g: \mathbb{R}^{n} \times \mathbb{R}^{m} \times \mathbb{R}^{s} \rightarrow \mathbb{R}^{r}$. We assume that there exists some $\varepsilon_{\max }>0$ and some compact set $\mathbb{Z}_{\max }$ such that for all $0 \leq|\varepsilon| \leq \varepsilon_{\max }$, the set $\mathbb{Z}_{\varepsilon}$ is non-empty and $\mathbb{Z}_{\varepsilon} \subseteq \mathbb{Z}_{\max }$. The sets of feasible and optimal steady-states can now be defined analogously to Section II-B, i.e., $S_{\varepsilon}:=\left\{(x, u) \in \mathbb{Z}_{\varepsilon}: x=f(x, u)\right\}$ and

$$
S_{\varepsilon}^{*}:=\left\{(y, w) \in S_{\varepsilon}: \ell(y, w)=\min _{(x, u) \in S_{\varepsilon}} \ell(x, u)\right\} .
$$

We assume that $S_{\varepsilon}$ (and hence also $S_{\varepsilon}^{*}$ ) are non-empty for all $0 \leq$ $|\varepsilon| \leq \varepsilon_{\max }$. As above, in the following $\left(x^{*}(\varepsilon), u^{*}(\varepsilon)\right)$ denotes an arbitrary element of $S_{\varepsilon}^{*}$. Note that $\left(x^{*}(\varepsilon), u^{*}(\varepsilon)\right)$ is a global minimizer of problem

$$
\mathcal{P}_{\ell}[\varepsilon]:=\mathcal{P}([x u], \ell, x-f(x, u), g)
$$

as defined in (21). The question we are interested in is under what circumstances there exists a storage function $\lambda(x ; \varepsilon)$ such that, if system (2) is dissipative with respect to the supply rate $s(x, u ; \varepsilon)=$ $\ell(x, u)-\ell\left(x^{*}(\varepsilon), u^{*}(\varepsilon)\right)$ for $\varepsilon=0$, it remains dissipative on $\mathbb{Z}_{\varepsilon}$ for changing $\varepsilon$. This means that the function

$$
\gamma(x, u ; \varepsilon):=\ell(x, u)-\ell\left(x^{*}(\varepsilon), u^{*}(\varepsilon)\right)+\lambda(x ; \varepsilon)-\lambda(f(x, u) ; \varepsilon)
$$

satisfies $\gamma(x, u ; \varepsilon) \geq 0$ for all $(x, u) \in \mathbb{Z}_{\varepsilon}$, i.e., $\left(x^{*}(\varepsilon), u^{*}(\varepsilon)\right)$ is a global minimizer of problem

$$
\mathcal{P}_{\gamma}[\varepsilon]:=\mathcal{P}\left(\left[\begin{array}{ll}
x & u
\end{array}\right], \gamma, 0, g\right)
$$

as defined in (21) with $\gamma\left(x^{*}(\varepsilon), u^{*}(\varepsilon) ; \varepsilon\right)=0$. The following Theorem shows under what conditions robustness of the dissipativity property with respect to small changes in $\varepsilon$ can be guaranteed.

Theorem 5: Suppose that the following is satisfied:

(i) The functions $f, \ell$ and $g$ are twice continuously differentiable in $(x, u)$. Furthermore, $g$ as well as its first and second derivatives with respect to $(x, u)$ are continuous in $\varepsilon$.

(ii) For $\varepsilon=0, S_{0}^{*}$ is a singleton, i.e., $\left(x^{*}(0), u^{*}(0)\right)$ is the unique optimal steady-state. Furthermore, $\left(x^{*}(0), u^{*}(0)\right)$ is the unique global minimizer of problem $\mathcal{P}_{\gamma}[0]$, i.e., system (2) is dissipative on $\mathbb{Z}_{0}$ with respect to the supply rate $s(x, u ; 0)=\ell(x, u)-$ $\ell\left(x^{*}(0), u^{*}(0)\right)$, and the corresponding storage function $\lambda(x ; 0)$ is twice continuously differentiable in $x$.

(iii) The optimal steady-state $\left(x^{*}(0), u^{*}(0)\right)$ is regular and satisfies the strong second order sufficiency condition (see $[20,21])$ for problems $\mathcal{P}_{\ell}[0]$ and $\mathcal{P}_{\gamma}[0]$.

Then there exists $\bar{\varepsilon}$ with $0<\bar{\varepsilon} \leq \varepsilon_{\max }$ such that for all $|\varepsilon| \leq \bar{\varepsilon}$ the system (2) is dissipative on $\mathbb{Z}_{\varepsilon}$ with respect to the supply rate $s(x, u ; \varepsilon)=\ell(x, u)-\ell\left(x^{*}(\varepsilon), u^{*}(\varepsilon)\right)$ and with storage function $\lambda(x ; \varepsilon):=\lambda(x ; 0)+\tilde{\lambda}(\varepsilon)^{T} x$, where $\tilde{\lambda}(\varepsilon)$ is continuous in $\varepsilon$ with $\tilde{\lambda}(0)=0$.

Theorem 5 means that the storage function $\lambda(x ; \varepsilon)$ can be modified continuously with changing parameters $\varepsilon$ such that the system remains dissipative with respect to the supply rate $s(x, u ; \varepsilon)=$ $\ell(x, u)-\ell\left(x^{*}(\varepsilon), u^{*}(\varepsilon)\right)$. The proof of Theorem 5 , which can be found in Section II.A of the technical report [14], uses the sensitivity analysis in nonlinear programming [20-22] to conclude that under the given assumptions, the minimizer of problem $\mathcal{P}_{\ell}[\varepsilon]$ is continuous in $\varepsilon$ for small $|\varepsilon|$. Then, it is shown that the storage function $\lambda(x ; \varepsilon)$ can be modified continuously in $\varepsilon$ such that the minimizer of problem $\mathcal{P}_{\gamma}[\varepsilon]$ coincides with the minimizer of problem $\mathcal{P}_{\ell}[\varepsilon]$. In particular, the (in general nonlinear) storage function $\lambda$ can be modified by an additional linear term $\tilde{\lambda}(\varepsilon)^{T} x$ in order to still serve as a storage function for the system under changing constraints.

Remark 7: In view of Theorem 1, with the help of Theorem 5 one can ensure that system (2) is robustly optimally operated at steadystate with respect to small changes in the state and input constraints. $\square$

Remark 8: The results of Theorem 5 can be extended in a straightforward way to the case where also the stage cost function $\ell$ and the system dynamics $f$ depend on the additional parameters $\varepsilon$, i.e., robustness of dissipativity with respect to small changes in the cost function and the system dynamics can be established. Furthermore, the results of Theorem 5 can also be extended to an economic MPC setting including average constraints, i.e., constraints on average quantities of input and state variables. For such a setting, it was shown in $[4$, Section V.B] that a relaxed dissipativity condition involving an additional free multiplier in the supply rate is sufficient for optimal steady-state operation. Similar to the proof of Theorem 5, one can show that both the storage function $\lambda$ and this multiplier can be modified continuously in $\varepsilon$ such that dissipativity is maintained under small changes in $\varepsilon$. Finally, we note that the presented robustness results can be extended to the case of general parameter dependent supply rates $s(x, u ; \varepsilon)$, different from the specific one considered above, and hence might be of interest also beyond an economic MPC context. This is shown in more detail in Section III of the technical report [14].

\section{B. Convex case}

In this section, we show that further results beyond the robustness analysis of Section IV-A can be obtained if a certain convexity assumption is satisfied. Namely, instead of considering small perturbations in the constraint set as in Section IV-A (which were expressed by the parameter $\varepsilon$ ), we now look at additional constraints $g_{a d}$ which are imposed on the system and can alter the optimal steady-state to a large extend. Hence in the following, we drop the dependence of the various functions and optimization problems on $\varepsilon$, but instead define the sets $\mathbb{Z}_{a d}:=\left\{(x, u): g(x, u) \leq 0, g_{a d}(x, u) \leq 0\right\}$, $S_{a d}:=\left\{(x, u) \in \mathbb{Z}_{a d}: x=f(x, u)\right\}$ and $S_{a d}^{*}:=\left\{(y, w) \in S_{a d}:\right.$ $\left.\ell(y, w)=\min _{(x, u) \in S_{a d}} \ell(x, u)\right\}$. Analogous to above, let $\left(x_{a d}^{*}, u_{a d}^{*}\right)$ denote an arbitrary element of $S_{a d}^{*}$, and define the optimization problems $\mathcal{P}_{\ell, a d}:=\mathcal{P}\left(\left[\begin{array}{ll}x & u\end{array}\right], \ell, x-f(x, u),\left[\begin{array}{ll}g & g_{a d}\end{array}\right]\right)$ and $\mathcal{P}_{\gamma_{a d}}:=$ $\mathcal{P}\left(\left[\begin{array}{ll}x u\end{array}\right], \gamma_{a d}, 0,\left[\begin{array}{ll}g & g_{a d}\end{array}\right]\right)$ with

$$
\gamma_{a d}(x, u):=\ell(x, u)-\ell\left(x_{a d}^{*}, u_{a d}^{*}\right)+\lambda_{a d}(x)-\lambda_{a d}(f(x, u))
$$

for some storage function $\lambda_{a d}(x)$. The following Theorem shows that if $\gamma$ as defined in (12) is convex, then for each feasible steady-state $(y, w) \in S$ there exists a function $g_{a d}$ such that $(y, w)$ is an optimal steady-state under the additional constraints, i.e., $(y, w) \in S_{a d}^{*}$, and dissipativity with respect to the new supply rate $s_{a d}(x, u)=\ell(x, u)-$ $\ell\left(x_{a d}^{*}, u_{a d}^{*}\right)$ is maintained with the same storage function $\lambda_{a d}(x)=$ $\lambda(x)$.

Theorem 6: Suppose that the following is satisfied:

(i) The functions $f, g$ and $\ell$ are continuously differentiable in $(x, u)$ and $g$ is convex.

(ii) There exist a continuously differentiable storage function $\lambda(x)$ such that system (2) without additional constraints is dissipative on $\mathbb{Z}$ with respect to the supply rate $s(x, u)=\ell(x, u)-$ 
$\ell\left(x^{*}, u^{*}\right)$, and the corresponding function $\gamma$ defined in (12) is convex on $\mathbb{Z}$.

Then, for each feasible steady-state $(y, w) \in S$, there exists an additional constraint function $g_{a d}$ which is convex and continuously differentiable in $(x, u)$ such that $(y, w) \in S_{a d}^{*}$ and the system (2) is dissipative on $\mathbb{Z}_{a d}$ with respect to the supply rate $s_{a d}(x, u)=$ $\ell(x, u)-\ell\left(x_{a d}^{*}, u_{a d}^{*}\right)$ and with storage function $\lambda_{a d}(x)=\lambda(x)$.

Conversely, if for a given convex and continuously differentiable additional constraint function $g_{a d}$, a steady-state $(y, w) \in S$ together with some $\nu=\left[\begin{array}{ll}\nu_{g_{a d}}^{T} & \nu_{g}^{T}\end{array}\right]^{T}$ satisfies the Karush-Kuhn-Tucker (KKT) conditions (see, e.g., [23]) for problem $\mathcal{P}_{\gamma, a d}$ with $\lambda_{a d}(x)=\lambda(x)$, then $(y, w) \in S_{a d}^{*}$ and system (2) is dissipative on $\mathbb{Z}_{a d}$ with respect to the supply rate $s_{a d}(x, u)=\ell(x, u)-\ell\left(x_{a d}^{*}, u_{a d}^{*}\right)$ and with storage function $\lambda_{a d}(x)=\lambda(x)$.

Proof: The proof of Theorem 6 can be found in Section II.B of the technical report [14].

Remark 9: The convexity assumption on $\gamma$ in (ii) is always satisfied if $\ell$ is strictly convex and system (2) is linear. In this case, if Slater's condition is satisfied, strong duality holds and the storage function $\lambda$ in (12) can be chosen as a linear function [10]. However, note that for $\gamma$ to be convex, neither $\ell$ has to be convex nor the system (2) has to be linear.

Remark 10: If $\gamma$ is not convex, the statements of Theorem 6 still hold for each steady-state $(y, w) \in S$ such that $\gamma$ can be lower bounded on $\mathbb{Z}$ by a convex and continuously differentiable function $\hat{\gamma}$ satisfying $\hat{\gamma}(y, w)=\gamma(y, w)$. Namely, as $\hat{\gamma}$ is convex, one can establish as in the proof of Theorem 6 that $(y, w)$ minimizes $\hat{\gamma}$ over $\mathbb{Z}_{a d}$. But then, as $\hat{\gamma}$ is a lower bound for $\gamma$ on $\mathbb{Z}$ and furthermore $\hat{\gamma}(y, w)=\gamma(y, w)$, it follows that $(y, w)$ also minimizes $\gamma$ (and hence also $\gamma_{a d}$ ) over $\mathbb{Z}_{a d}$, i.e., the system is again dissipative on $\mathbb{Z}_{a d}$ with respect to the supply rate $s_{a d}(x, u)=\ell(x, u)-\ell\left(x_{a d}^{*}, u_{a d}^{*}\right)$ and with storage function $\lambda_{a d}(x)=\lambda(x)$.

\section{CONCLUSions}

In this paper, we investigated a dissipativity condition which was recently used in several papers on economic MPC in order to establish optimal steady-state operation as well as convergence and stability of the closed-loop system. As a first main contribution, we established two (partial) converse theorems showing that dissipativity is in fact necessary for optimal steady-state operation, given that a certain controllability condition is satisfied, which means that dissipativity is a precise characterization of optimal steady-state operation and not only a rather conservative sufficient condition for it. Furthermore, the obtained results are also of importance in the context of stability analysis of economic MPC, as there typically the existence of a storage function $\lambda$ is sufficient but $\lambda$ does not have to be known. Second, we provided a robustness analysis of the dissipativity property with respect to changes in the constraint set. We showed that under a certain regularity assumption, robustness with respect to small changes in the constraints can be guaranteed. Furtermore, stronger results on maintaining the dissipativity property under possibly large changes in the constraints were obtained given that a certain convexity assumption is satisfied.

\section{REFERENCES}

[1] M. A. Müller and F. Allgöwer, "Robustness of steady-state optimality in economic model predictive control," in Proceedings of the 51st IEEE Conference on Decision and Control, 2012, pp. 1011-1016.

[2] J. B. Rawlings and D. Q. Mayne, Model Predictive Control: Theory and Design. Madison, WI: Nob Hill Publishing, 2009.

[3] L. Grüne and J. Pannek, Nonlinear Model Predictive Control. Theory and Algorithms. London: Springer, 2011.
[4] D. Angeli, R. Amrit, and J. B. Rawlings, "On average performance and stability of economic model predictive control," IEEE Transactions on Automatic Control, vol. 57, no. 7, pp. 1615-1626, 2012.

[5] T. G. Hovgaard, L. F. S. Larsen, and J. B. Jørgensen, "Flexible and cost efficient power consumption using economic MPC - a supermarket refrigeration benchmark," in Proceedings of the 50th IEEE Conference on Decision and Control and European Control Conference, 2011, pp. 848-854.

[6] R. Huang, E. Harinath, and L. T. Biegler, "Lyapunov stability of economically oriented NMPC for cyclic processes," Journal of Process Control, vol. 21, no. 4, pp. 501-509, 2011.

[7] W.-J. Ma and V. Gupta, "Desynchronization of thermally-coupled firstorder systems using economic model predictive control," in Proceedings of the 51st IEEE Conference on Decision and Control, 2012, pp. 278283.

[8] J. B. Rawlings, D. Bonné, J. B. Jørgensen, A. N. Venkat, and S. B. Jørgensen, "Unreachable setpoints in model predictive control," IEEE Transactions on Automatic Control, vol. 53, no. 9, pp. 2209-2215, 2008.

[9] D. Angeli and J. B. Rawlings, "Receding horizon cost optimization and control for nonlinear plants," in Proceedings of the 8th IFAC Symposium on Nonlinear Control Systems, 2010, pp. 1217-1223.

[10] M. Diehl, R. Amrit, and J. B. Rawlings, "A Lyapunov function for economic optimizing model predictive control," IEEE Transactions on Automatic Control, vol. 56, no. 3, pp. 703-707, 2011.

[11] R. Amrit, J. B. Rawlings, and D. Angeli, "Economic optimization using model predictive control with a terminal cost," Annual Reviews in Control, vol. 35, no. 2, pp. 178-186, 2011.

[12] M. Heidarinejad, J. Liu, and P. D. Christofides, "Economic model predictive control of nonlinear process systems using Lyapunov techniques," AIChE Journal, vol. 58, no. 3, pp. 855-870, 2012.

[13] L. Grüne, "Economic receding horizon control without terminal constraints," Automatica, vol. 49, no. 3, pp. 725-734, 2013.

[14] M. A. Müller, D. Angeli, and F. Allgöwer, "Additional material to the paper 'On necessity and robustness of dissipativity in economic model predictive control'," University of Stuttgart, Tech. Rep., 2014, available online: www.simtech.uni-stuttgart.de/publikationen/prints.php?ID=903.

[15] J. C. Willems, "Dissipative dynamical systems - part i: General theory," Archive for Rational Mechanics and Analysis, vol. 45, no. 5, pp. 321$351,1972$.

[16] C. I. Byrnes and W. Lin, "Losslessness, feedback equivalence, and the global stabilization of discrete-time nonlinear systems," IEEE Transactions on Automatic Control, vol. 39, no. 1, pp. 83-98, 1994.

[17] M. A. Müller, D. Angeli, and F. Allgöwer, "On convergence of averagely constrained economic MPC and necessity of dissipativity for optimal steady-state operation," in Proceedings of the American Control Conference, 2013, pp. 3147-3152.

[18] E. D. Sontag, Mathematical Control Theory - Deterministic Finite Dimensional Systems, 2nd ed. New York: Springer, 1998.

[19] T. Damm, L. Grüne, M. Stieler, and K. Worthmann, "An exponential turnpike theorem for dissipative discrete time optimal control problems," SIAM Journal on Control and Optimization, vol. 52, no. 3, pp. 19351957, 2014.

[20] K. Jittorntrum, "Solution point differentiability without strict complementarity in nonlinear programming," in Sensitivity, Stability and Parametric Analysis, ser. Mathematical Programming Studies, A. V. Fiacco, Ed. Springer Berlin Heidelberg, 1984, vol. 21, pp. 127-138.

[21] S. M. Robinson, "Strongly regular generalized equations," Mathematics of Operations Research, vol. 5, no. 1, pp. 43-62, 1980.

[22] A. V. Fiacco and Y. Ishizuka, "Sensitivity and stability analysis for nonlinear programming," Annals of Operations Research, vol. 27, no. 1, pp. 215-235, 1990.

[23] D. P. Bertsekas, Nonlinear Programming. Belmont, Massachusetts: Athena Scientific, 1995. 